\title{
Applications of Fuzzy adaptive PID control in the thermal power plant denitration liquid ammonia evaporation
}

\author{
Jing $\mathrm{Li}^{1}$, Guo Qing Zhang ${ }^{2}$, Huan Xin Cheng ${ }^{3, a}$,Li Cheng ${ }^{4, a}$ \\ 1,2,3 College of Automation and Electrical Engineering. Qingdao University of Science and Technology. Qingdao 266042.china \\ ${ }^{4}$ Xinjiang Technical Institute of Physics and Chemistry.CAS, Urumuqi,Xinjiang, 830011, China \\ a Urumqi Xiaming Innovation Electronic Technology Co.,Ltd,Urumuqi,Xinjiang, 830011,China
}

\begin{abstract}
For the control of the liquid level of liquid ammonia in thermal power plant's ammonia vaporization room, traditional PID controller parameter tuning is difficult to adapt to complex control systems, the setting of the traditional PID controller parameters is difficult to adapt to the complex control system. For the disadvantage of bad parameter setting, poor performance and so on, the fuzzy adaptive PID control is proposed. Fuzzy adaptive PID control combines the advantages of traditional PID technology and fuzzy control. By using the fuzzy controller to intelligent control the object, the performance of the PID controller is further improved, and the control precision of the system is improved ${ }^{[1]}$. The simulation results show that the fuzzy adaptive PID controller not only has the advantages of high accuracy of PID controller, but also has the characteristics of fast and strong adaptability of fuzzy controller. It realizes the optimization of PID parameters which are in the optimal state, and the maximum increase production efficiency, so that are more suitable for nonlinear dynamic system.
\end{abstract}

\section{Introduction}

Coal is the main energy source of our country nowadays and the future. When coal is burned in the boiler, sulfur and nitrogen oxide waste gas can be generated. Nitrogen oxides are one of the key reasons for the regional air pollution. It is not only the cause of acid rain and fog, but also causes the other mixed chemical air pollution, which is harmful to human survival and destruction of the ecological environment ${ }^{[2]}$. At present, although the vast majority of the thermal power plant is equipped with low nitrogen combustion technology in our country, but the denitration efficiency is low. The SCR technology combined with low nitrogen combustion technology using, out of stock will greatly increase the efficiency. Thermal power plant SCR device includes: flue gas system, ammonia injection system, liquid ammonia storage evaporation system, blowing ash system, SCR catalyst, and safety auxiliary equipment.

Liquid ammonia gasification is an important part of the thermal power plant denitration process hat ${ }^{[3]}$. It is also the first condition of ensure the normal operation of denitration. The safe operation of the liquid ammonia gasification directly affects the safety of the whole thermal power plant. Therefore to study advanced the application of DCS to control the denitration liquid ammonia vapors, improve the stability of the system is running safety is even more necessary, at the same time there is a growing concern of atmospheric pollution control is of great significance. So we put fuzzy adaptive
PID control in the process of liquid ammonia, improve the efficiency of liquid ammonia vaporization and to meet the requirements of safety and process constraints, making the key points of each control to achieve the optimization and for the denitration follow-up work well enough ammonia gas supply, ultimately achieve the denitration efficiency maximization.

\section{The design of fuzzy adaptive PID controller}

\subsection{PID control}

PID controller is a linear controller. As shown in figure 1,

$$
u(t)=k_{p} e(t)+k_{i} \int_{0}^{i} e(\tau) d \tau+\frac{d e(t)}{d t}
$$

In the formula, $k_{p}$ is the scale factor. $T_{i}$ is the integral time constant. $T_{d}$ is the differential time constant.

At present, the incremental PID control algorithm is widely used, and its output $\Delta u(k)$ is the increment of the control quantity. Its formula is as follows.

$$
\Delta u(k)=k_{p} \Delta e(k)+k_{i} e(k)+k_{d}[e(k)-\Delta e(k-1)]
$$

Corresponding author: 317842602@qq.com 


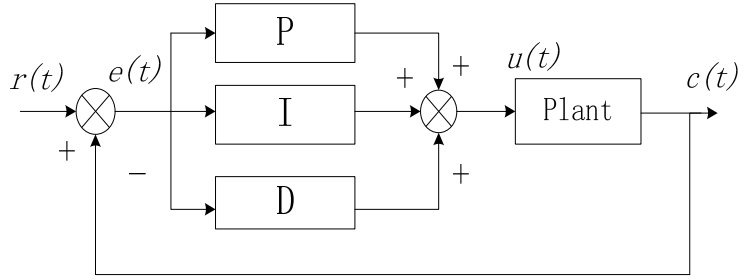

Figure 1. Block diagram of PID system

Among them, $K_{p}$ is the proportion coefficient; $K_{i}=K_{p} T / T_{i}$ is the integral coefficient; $K_{d}=K_{p} T_{d} / T$ is the differential coefficient.

In the PID control process, first determine the mathematical model based on the controlled object PID, and then use the deviation $\mathrm{e}$ as input to calculate the amount of control and drive the appropriate enforcement agency to reduce the error until the controlled object stabilized within the allowable range.

\subsection{The control principle of Fuzzy adaptive PID controller}

The control principle of fuzzy adaptive PID control is to identify the fuzzy relationship with PID three parameters $K_{p}, K_{i}, K_{d}$ between e and $\mathrm{e}_{\mathrm{c}}$, through continuous testing e and $e_{c}$ in the operation, based on fuzzy control theory to online modification of the three parameters in order to satisfy different $\mathrm{e}$ and $\mathrm{e}_{\mathrm{c}}$ for different requirements of the control parameters and leave the controlled object has a good dynamic and static performance ${ }^{[4]}$. The whole structure of fuzzy adaptive PID controller is as shown in figure 2 .

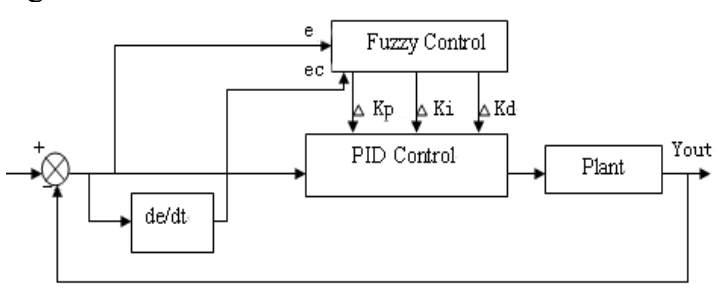

Figure 2. Block diagram of Fuzzy adaptive PID controller

\subsection{The design of fuzzy adaptive PID controller}

\subsubsection{The determination of membership function of linguistic variables}

We put the deviation e and the change rate deviation $e_{c}$ as the input fuzzy controller and the three parameters of PID controller $\Delta k_{p}, \Delta k_{i}, \Delta k_{d}$ as the output. According to the project of the actual situation, fuzzy subset of the input variables $\mathrm{e}$ and $\mathrm{e}_{\mathrm{c}}$ are \{negative big, negative middle, negative small, zero, positive small, positive middle, positive big\}, denoted as $\{\mathrm{NB}, \mathrm{NM}, \mathrm{NS}, \mathrm{ZO}, \mathrm{PS}$, $\mathrm{PM}, \mathrm{PB}\}$. The deviation e and deviation change rate $\mathrm{e}_{\mathrm{c}}$ were quantified into the region of $(-3,3)$. Meanwhile, the fuzzy subset of the output variable KP, KI, KD are \{negative big, negative middle, the negative small, zero, positive small, positive middle, positive big \} denoted as $\{\mathrm{NB}, \mathrm{NM}, \mathrm{NS}, \mathrm{ZO}, \mathrm{PS}, \mathrm{PM}, \mathrm{PB}\}$, respectively, to be quantified into the region of $(-3,3)(0.06,0.06)(0,3)$. These membership function curves are shown in figure 3 , figure 4.

According to the membership assignment table of each fuzzy subset and the fuzzy control model of each parameter, using fuzzy synthesis reasoning design the fuzzy matrix table of PID parameters to realize online correction PID parameters. Computation formula is as follows:

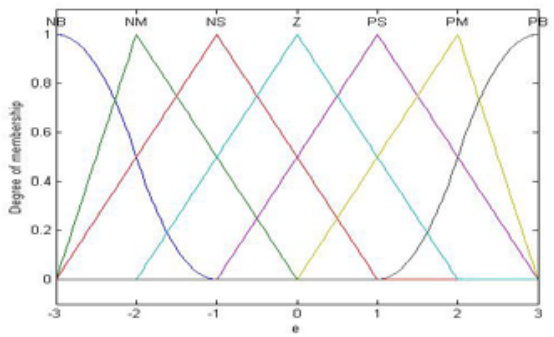

Figure 3. The membership function of input variable e $e_{c}$ curve
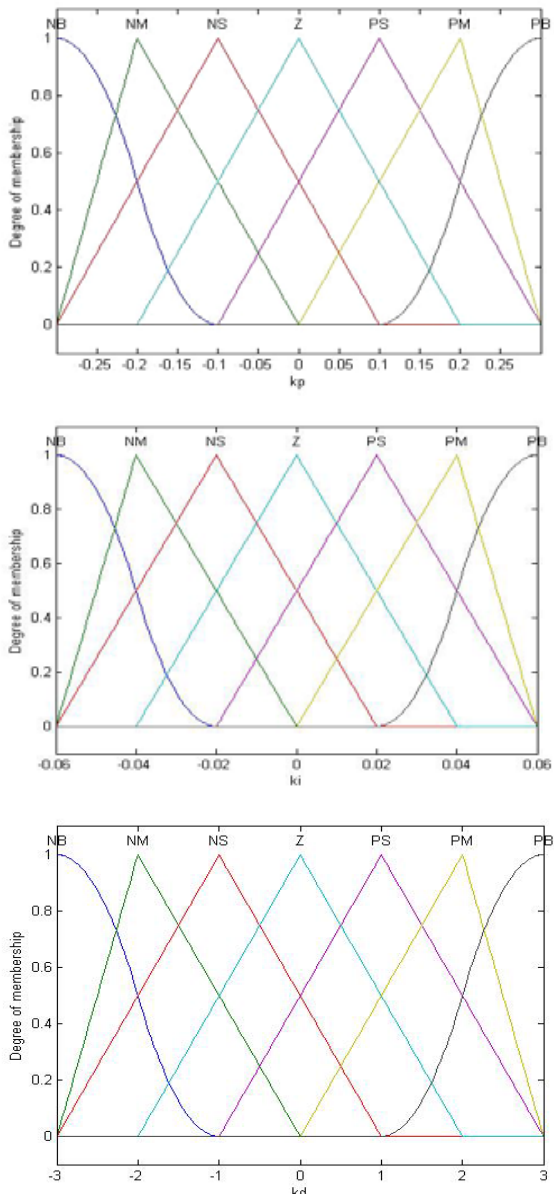

Figure 4. The membership function of output variable $\Delta k_{p} \Delta k_{i} \Delta k_{d}$

$$
\begin{aligned}
& k_{p}=k_{p}^{\prime}+\Delta k_{p} \\
& k_{i}=k_{i}^{\prime}+\Delta k_{i} \\
& k_{d}=k_{d}^{\prime}+\Delta k_{d}
\end{aligned}
$$


In the above formula $k_{p}^{\prime}, k_{i}^{\prime}, k_{d}^{\prime}$ is original PID parameter that have been setted .

\subsubsection{Control rule table of fuzzy controller}

Here's to establish the fuzzy rules with fuzzy adaptive PID control. First we need to judge the knowledge library based on expert knowledge and determine the three parameters in PID and fuzzy relationship between the deviation e and deviation change rate $e_{c}$. And then through continuous detection of the operation of $e$ and $e_{c}$, according to the fuzzy control rules to determine the three parameters to online adaptive in order to meet the different requirements of the two parameters of different e and $e_{c}$, leaving the controlled object has good dynamic and static performance.

According to the parameters $K_{p}, K_{i} K_{d}$ affect the output characteristics of the system, we can summarize the self-tuning principles of the system in the controlled process for different deviation e and deviation change rate $\mathrm{e}_{\mathrm{c}}$ and parameters $K_{p}, K,{ }_{i} K_{d}$ as follows:

1) When the deviation e is larger, in order to speed up the response speed of system and prevent deviation e at the beginning of the instant greatens may cause differential supersaturated and make the control action is beyond the scope of, we should take the larger KP and smaller KD, usually take $K_{i}=0$.

2) When the deviation e and deviation change rate $e_{c}$ are at medium size, in order to make the overshoot of system respond to reduce and guarantee a certain response speed, $K_{p}$ should take smaller. In this case, the value of $K_{d}$ has a great influence on the system, so we should also take a small number. The value of $K_{i}$ should be appropriate.

3) When the deviation e is smaller, in order to make the system have better steady-state performance, we should increase the value of $K_{p} \mathrm{KP}$ and $K_{i}$. At the same time in order to avoid output response's oscillation around the set value, and to consider anti-jamming capability of system, $K_{d}$ should be properly selected. Its principle is: when the deviation rate $e_{c}$ is smaller, the value of $K_{d}$ is larger, When the deviation rate $\mathrm{e}_{c}$ is larger, $K_{d}$ take smaller values; Usually $K_{d}$ for medium size.

Control rules table of output variable $\Delta k_{p} 、 \Delta k_{i}$ 、 $\Delta k_{d}$ as shown in table $1,2,3$.

Table 1. The fuzzy rule table of $\Delta k_{p}$

\begin{tabular}{|c|c|c|c|c|c|c|c|}
\hline $\mathrm{e}_{\mathrm{k}} \mathrm{e}_{\mathrm{f}}$ & $\mathrm{NB}$ & $\mathrm{NM}$ & $\mathrm{NS}$ & Z0 & PS & PM & PB \\
\hline NB & PB & PB & PM & PM & PS & Z0 & Z0 \\
NM & PB & PB & PM & PS & PS & Z0 & NS \\
NS & PM & PM & PM & PS & Z0 & NS & NS \\
Z0 & PM & PM & PS & Z0 & NS & NM & NM \\
PS & PS & PS & Z0 & NS & NS & NM & NM \\
\hline
\end{tabular}

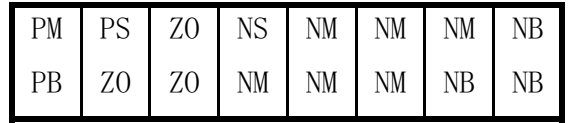

Table 2. The fuzzy rule table of $\Delta k_{i}$

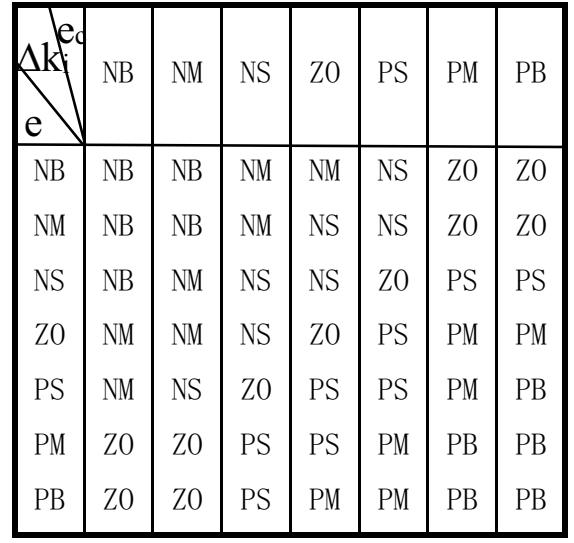

Table 3. The fuzzy rule table of $\Delta k_{d}$

\begin{tabular}{|l|l|l|l|l|l|l|l|}
\hline Ake & NB & NM & NS & Z0 & PS & PM & PB \\
\hline e & & & & & & \\
\hline NB & PS & NS & NB & NB & NB & NM & PS \\
NM & PS & NS & NB & NM & NM & NS & Z0 \\
NS & Z0 & NS & NM & NM & NS & NS & Z0 \\
Z0 & Z0 & NS & NS & NS & NS & NS & Z0 \\
PS & Z0 & Z0 & Z0 & Z0 & Z0 & Z0 & Z0 \\
PM & PB & NS & PS & PS & PS & PS & PB \\
PB & PB & PM & PM & PM & PS & PS & PB \\
\hline
\end{tabular}

\subsubsection{Control rule table of fuzzy controller}

Mamdani inference method is a common method used in fuzzy control. Using a fuzzy relation by $\mathrm{X}$ to $\mathrm{Y}$ to represent a fuzzy conditional statement: if $\mathrm{A}$ then $\mathrm{B}$, When the input is $\mathrm{A}^{*}, \mathrm{~B}^{*}$ can be obtained from the output.

$$
B^{*}=A^{*} \circ R_{a \rightarrow b}
$$

It represents the synthesis operation of fuzzy relations.

Mamdani fuzzy relationship definition of $\mathrm{R}_{\mathrm{a}} \rightarrow \varepsilon$ based on

$$
u_{R_{A \rightarrow B}}(x, y)=\left[u_{A}(x) \wedge u_{B}(y)\right]
$$

Therefore, according to the membership function of controller's output B * is

$$
\mu_{B^{*}}(y)=\sup _{x \in X}\left\{\mu_{A^{*}}(x) \wedge\left[\mu_{A}(x) \wedge \mu_{B}(y)\right]\right\}
$$

\subsubsection{Defuzzification}

The process of converting the fuzzy quantity into the precise quantity is called as the clarity, also known as the fuzzy, or called the fuzzy judgment. In order to obtain the accurate controlled variable, the fuzzy method is required to be able to output the accurate calculation results of the membership function. In this paper, we use the fuzzy method widely used in industrial control, 
weighted average method, is taking a weighted average of the membership degree of output value clearly. Assuming that the output fuzzy sets can be represented as $U=\sum u_{U}\left(x_{i}\right) /\left(x_{i}\right)$, according to the following formula, we can clear quantity as follows:

$$
u_{c}=\frac{\int_{U} x u_{U}(x) d x}{\int_{U} u_{U}(x) d x}
$$

\section{System simulation using Simulink}

The fuzzy adaptive PID controller is used for liquid ammonia buffer tank level control. Simulink program of Fuzzy PID liquid ammonia buffer tank liquid level adaptive control is as shown below. Pay attention to the deviation e and its change size when the controlled object is in the actual passing ammonia. After the fuzzification we need check out the $\Delta k_{p}, \Delta k_{i}, \Delta k_{d}$ on the above should be in line, and then multiplied by the formula according to the respective ratio coefficient. Then use the formula to calculate the dynamic online PID parameters.

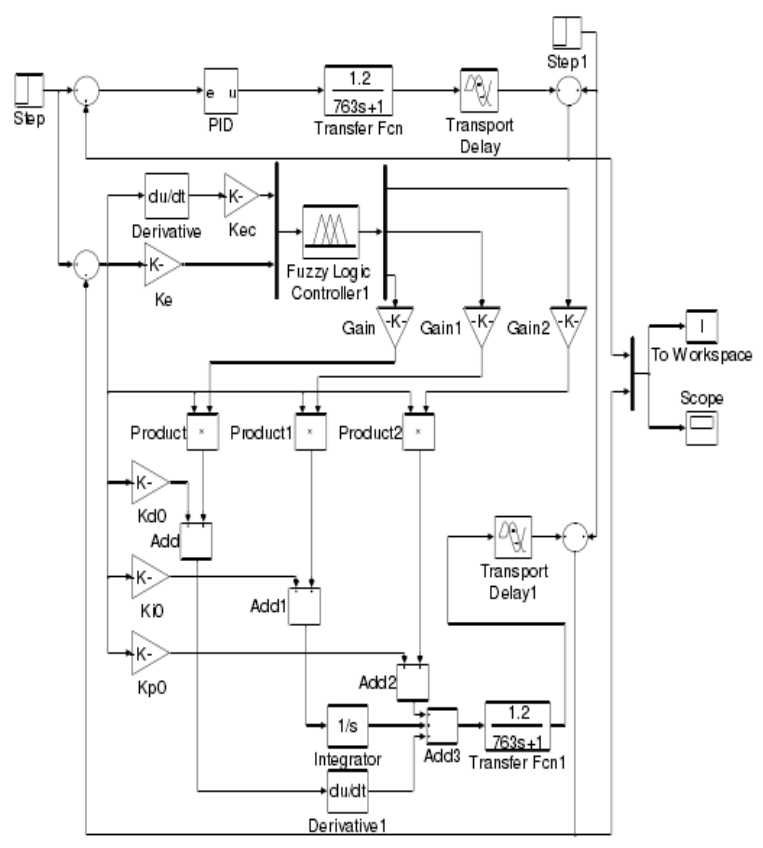

Figure 5. Fuzzy Adaptive PID Liquid Ammonia Buffer Tank Liquid Level Control System Simulation

Figures and tables, as originals of good quality and well contrasted, are to be in their final form, ready for reproduction, pasted in the appropriate place in the text. Try to ensure that the size of the text in your figures is approximately the same size as the main text (10 point). Try to ensure that lines are no thinner than 0.25 point.

At the beginning of setting parameters we use the experience of the actual project, assuming that the initial level is 1 . When the simulation time is 5000 seconds, the step disturbance that value is 0.2 was added. The total simulation time is 10,000 seconds. Simulation type selects ode5 fixed step size and step length value is 1 . In the fuzzy adaptive liquid ammonia buffer tank liquid level control system, deviation value $K_{e}$ is 0.1 , deviation change values $K_{e c}$ is 20 , object Online proportional gain quantization factor is 4.5 , integral gain quantization factor is 0.0045 , the initial values of proportional, integral, differential are $0.8,0.0012,50^{[5]}$. According to the formula, it can be concluded that the values of actual control ratio coefficient, the integral time and the differential time. The step response curve of fuzzy adaptive PID liquid ammonia buffer tank control system is obtained according to the simulation program. As shown in the figure below ${ }^{[6]}$.

The transfer function of the liquid ammonia buffer tank level control is as follow.

$$
G_{(S)}=\frac{7.3118}{500 s+1}
$$

Step response curve of fuzzy PID ammonia buffer tank level control system is shown below.

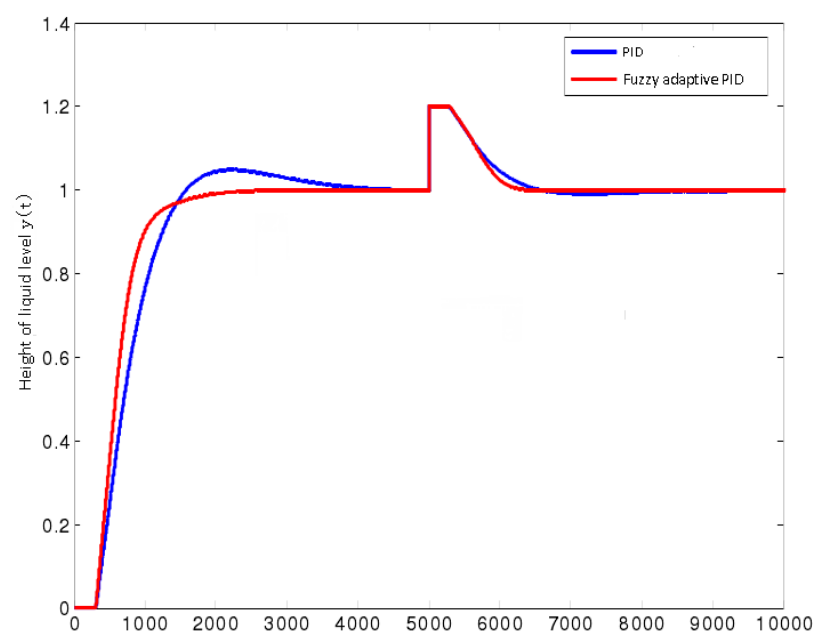

Figure 6. Traditional PID and Fuzzy Adaptive PID Algorithm Level Control Simulation Curve

Through the simulation, we can see that the fuzzy adaptive PID can reach the preset liquid level more quickly. That is to say, compared to the traditional PID, it has faster rising speed, shorter time. And the traditional PID control has overshoot, fuzzy control PID without overshoot, so that it can be very effective to reduce the impact of hysteresis ${ }^{[7]}$. In the $5000 \mathrm{~s}$, we give controlled object step disturbance of value is 0.2 . Because in the actual engineering projects the collected signal is often affected by human being unable to control the interference signal, so when adding disturbance we observed will be more in line with the real situation. We can also see that the fuzzy control PID has better robustness and anti- disturbance ability compared with the traditional PID control ${ }^{[8]}$. This is because the control algorithm can adjust the PID parameters according to the deviation and deviation change rate and make the proportional, integral and differential parameters in 
algorithm were within reasonable range. As seen in the simulation, the response can more quickly return to the set value, so that the fuzzy adaptive PID control has better effect ${ }^{[9]}$.

\section{Conclusions}

In this paper, through the establishment of fuzzy adaptive PID controller to control the liquid ammonia system, and simulation was carried out using Simulink ${ }^{[10]}$. The simulation results show that the fuzzy adaptive PID control compared with the traditional PID control has great superiority. It possesses the advantages of both PID control and fuzzy control, which shortened the time stability and reduce the overshoot. Fuzzy adaptive PID control has strong robustness and adaptive ability. When there is an outside interference, its control effect is satisfactory.

\section{References}

1. A.S. Hodel, C.E.Hall.Variable-structure PID Control to prevent integrator windup[J]. IEEE Transactions on Industrial Electronics, 2001, 48(2): 442 451.

2. F.De Lillo, F.Cecconi, G.Lacorata, A.Vulpiani, EPL, 84 (2008)

3. Kennedy J, Eberhart R C, Particle swarm optimization[J], Proc. IEEE International Conf. on Neural Networks, Perth, (8): IEEE Piscataway, 1995: 1942-1948.

4. Zhongcai Li, Wushan Cheng, Application of fuzzy PID control algorithm in ground source heat pump air conditioning control[J], Journal of Shanghai University of Engineering Science, 2012, 44(3): 215218.

5. Runyu Ye, A fuzzy adaptive PID control system computer simulation[J]. Information technology, 2007, (1): 801-521.

6. Ming Su, et al. Fuzzy PID control and its MATLAB simulation[J]. computer applications, 2004,(4).

7. Dingyu Xue, Yangquan Cheng, System simulation technology and application based on MATLAB/Simulink[M] 2 version, Beijing: Tsinghua university press, 2011.

8. Wei Lou, Xiangdong Liu. Application of fuzzy control in the boiler drum water level control system[J]. Boiler Technology. 2010, 41(1): 27-30.

9. Dong Yang, Experimental study on the formation of nitrogen oxides in coal combustion [D], [master's thesis] Shandong: Shan Dong university, 2004.

10. Guoguang Tian, Online self-learning of fuzzy controller rules[D], [master's thesis] Beijing, Beijing University of Chemical Technology, 2003. 